\title{
A serum-stable branched dimeric anti-VEGF peptide blocks tumor growth via anti-angiogenic activity
}

\author{
Jung-Wook Kim${ }^{1}$, Tae-Dong Kim², Bok Sil Hong', \\ Oh Youn Kim ${ }^{1}$, Wan-Hee Yoon ${ }^{2}$, Chi-Bom Chae ${ }^{3}$ and \\ Yong Song Gho ${ }^{1,4}$
}

\author{
${ }^{1}$ Department of Life Science \\ Division of Molecular and Life Sciences \\ Pohang University of Science and Technology \\ Pohang 790-784, Korea \\ ${ }^{2}$ Department of Biochemistry \\ College of Medicine \\ Chungnam National University \\ Daejeon 301-130, Korea \\ ${ }^{3}$ Department of Biomedical Science and Technology \\ Institute of Biomedical Science and Technology \\ Konkuk University \\ Seoul 143-701, Korea \\ ${ }^{4}$ Corresponding author: Tel, 82-54-279-2345; \\ Fax, 82-54-279-8609; E-mail, ysgho@ postech.ac.kr \\ DOI 10.3858/emm.2010.42.7.052
}

Accepted 8 June 2010

Available Online 14 June 2010

Abbreviations: bFGF, basic fibroblast growth factor; HUVEC, human umbilical vein endothelial cell; MAP, multiple antigenic peptide; VEGFR, vascular endothelial growth factor receptor

\begin{abstract}
Angiogenesis is critical and indispensable for tumor progression. Since VEGF is known to play a central role in angiogenesis, the disruption of VEGF-VEGF receptor system is a promising target for anti-cancer therapy. Previously, we reported that a hexapeptide (RRKRRR, RK6) blocked the growth and metastasis of tumor by inhibiting VEGF binding to its receptors. In addition, dRK6, the D-form derivative of RK6, retained its biological activity with improved serum stability. In the present study, we developed a serum-stable branched dimeric peptide (MAP2-dRK6) with enhanced anti-VEGF and anti-tumor activity. MAP2-dRK6 is more effective than dRK6 in many respects: inhibition of VEGF binding to its receptors, VEGF- and tumor conditioned medium-induced proliferation and ERK signaling of endothelial cells, and VEGF-induced migration and tube formation of endothelial cells. Moreover, MAP2-dRK6 blocks in vivo growth of
\end{abstract}

VEGF-secreting colorectal cancer cells by the suppression of angiogenesis and the subsequent induction of tumor cell apoptosis. Our observations suggest that MAP2-dRK6 can be a prospective therapeutic molecule or lead compound for the development of drugs for various VEGF-related angiogenic diseases.

Keywords: angiogenesis inhibitors; colorectal neoplasms; peptides; receptors, vascular endothelial growth factor; vascular endothelial growth factors

\section{Introduction}

Angiogenesis, the sprouting of new blood vessels from pre-existing vasculature, occurs during embryonic development and physiological processes such as wound healing and formation of the female reproductive system in a highly controlled manner by coordination of various pro- and anti-angiogenic factors (Liotta et al., 1991; Carmeliet, 2000). However, the balance between these factors is lost in pathological conditions like rheumatoid arthritis, diabetic retinopathy, and especially cancer, resulting in abnormal and inadequate growth of blood vessels (Carmeliet and Jain, 2000). Among numerous pro-angiogenic factors, VEGF is known to be specific to endothelial cells and critical for angiogenesis (Yancopoulos et al., 2000). VEGF is a homodimeric glycoprotein that exerts its activity via binding to two receptors, VEGF receptor1 (VEGFR1) and VEGFR2, expressed on the surface of endothelial cells (de Vries et al., 1992; Millauer et al., 1993). There are substantial evidences that VEGF is a major factor in tumor angiogenesis (Millauer et al., 1993; Kondo et al., 1994). Therefore, anti-angiogenic approaches for cancer treatment by disrupting VEGF-VEGFR system have been attempted (Kim et al., 1992; Aiello et al., 1995; Borgstrom et al., 1996; Strawn et al., 1996).

We previously reported that a VEGF-binding hexapeptide (RRKRRR, RK6) identified by screening peptide library antagonizes the activity of VEGF, resulting in the inhibition of the growth and metastasis of VEGF-secreting tumor (Bae et al., 2000). We then identified dRK6 (rrkrrr), a derivative of RK6 with D-amino acids having increased stability in serum and similar anti-VEGF activity as RK6 (Yoo et al., 2005). However, because of low anti-VEGF activity of RK6 and dRK6, the develop- 
A<smiles>N=C(N)NCCC[C@H](N)C(=O)N[C@@H](CCCNC(=N)N)C(=O)N[C@@H](CCCCN)C(=O)N[C@@H](CCCNC(=N)N)C(=O)N[C@@H](CCCNC(=N)N)C(=O)N[C@@H](CCCNC(=N)N)C(=O)O</smiles>

$\mathrm{NH}_{2}-\mathrm{RRKRRR}$

C

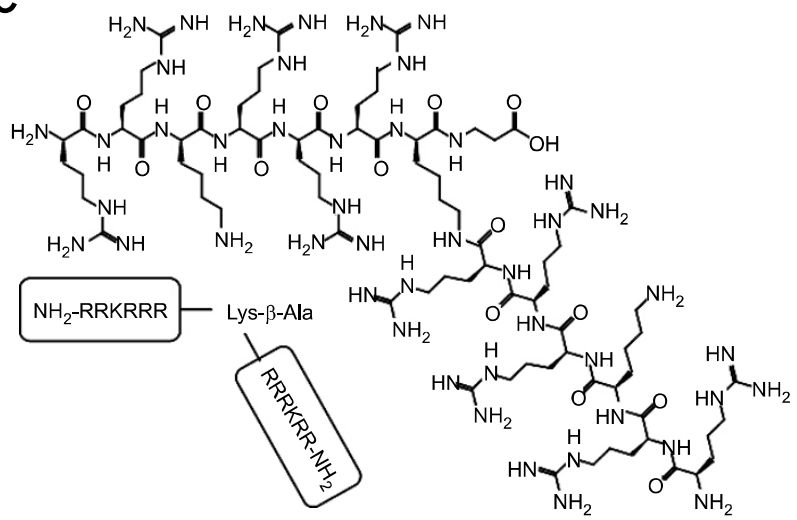

B<smiles>N=C(N)NCCCCC(NC(=O)C(CCCCNC(N)=O)NC(=O)C(CCCCN)NC(=O)C(CCCCN)NC(=O)C(N)CCCNC(N)=O)C(=O)NC(CCCCN)C(=O)O</smiles>

$\mathrm{NH}_{2}$-rrkrrr

D

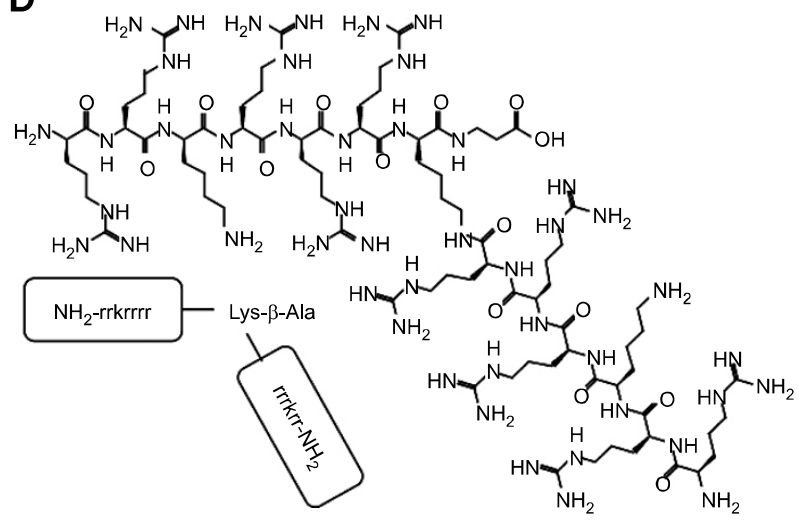

Figure 1. Structures of RK6, dRK6, MAP2-RK6, and MAP2-dRK6. (A) RK6 (RRKRRR). (B) dRK6 (rrkrrr), an RK6 derivative composed of D-amino acids MAP2-RK6 (C) and MAP2-dRK6 (D) are branched dimeric peptides with two RK6 and two dRK6 peptides, respectively, linked to $\alpha$-amino group and $\varepsilon$-amino group of lysine in the lysine- $\beta$-alanine branching unit.

ment of more potent VEGF-antagonizing peptides with enhanced serum stability was necessary for effective inhibition of angiogenesis. Several modifications have been introduced to increase the activity and stability of various peptides (Sahu et al., 2000; Lee et al., 2005). Among the various modification approaches, multiple antigenic peptide (MAP) system has been used to enhance the potency of peptides (Nomizu et al., 1993; Iwamoto et al., 1996; Sadler and Tam, 2002). MAP is a multimeric peptide derivative in which several peptide molecules are assembled on the branches of a lysine tree (Tam, 1988). The core lysine is located in the middle allowing multimer of active peptides to be accessible for efficient interactions.

In this study, we developed MAP2-dRK6, a branched dimeric peptide with two dRK6 peptides linked to $\alpha$-amino group and $\varepsilon$-amino group of lysine in the lysine- $\beta$-alanine branching unit. We observed that MAP2-dRK6 more effectively inhibited in vitro angiogenesis and in vivo tumor angiogenesis and subsequent tumor growth than dRK6 through the enhanced anti-VEGF activity.
These results suggest that MAP2-dRK6 may be a potential anti-VEGF drug candidate for targeting angiogenesis in many VEGF-related disorders.

\section{Results}

\section{Serum-stable MAP2-dRK6 has more potent anti-VEGF activity than RK6 and dRK6}

In our previous reports, a VEGF-binding hexapeptide RK6 inhibited the binding of VEGF to its receptors (Bae et al., 2000), and dRK6, its derivative composed of $\mathrm{D}$-amino acids, showed increased serum stability with similar activity in the inhibition of VEGF binding to receptors (Yoo et al., 2005). To develop more potent anti-VEGF peptides with enhanced serum stability, we first synthesized four peptides, RK6, dRK6, MAP2-RK6, and MAP2-dRK6 (Figure 1). MAP2-RK6 and MAP2-dRK6 are branched dimeric peptides with two RK6 and two dRK6 peptides, respectively, linked to $\alpha$-amino group and $\varepsilon$-amino group of 
A

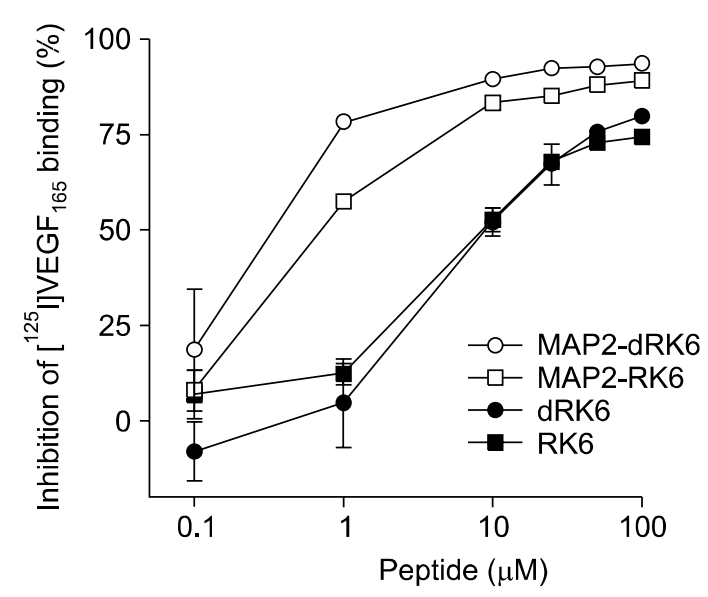

B

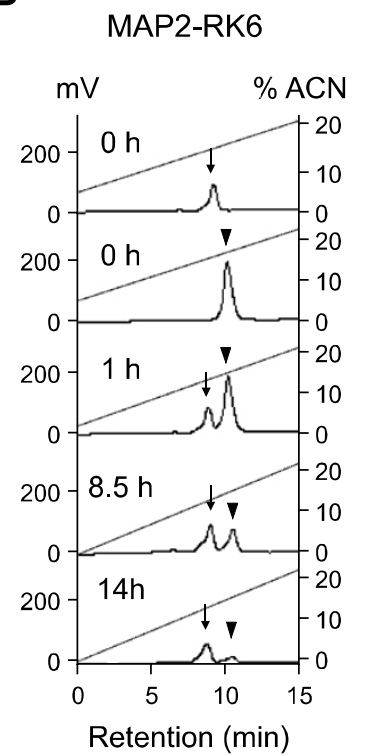

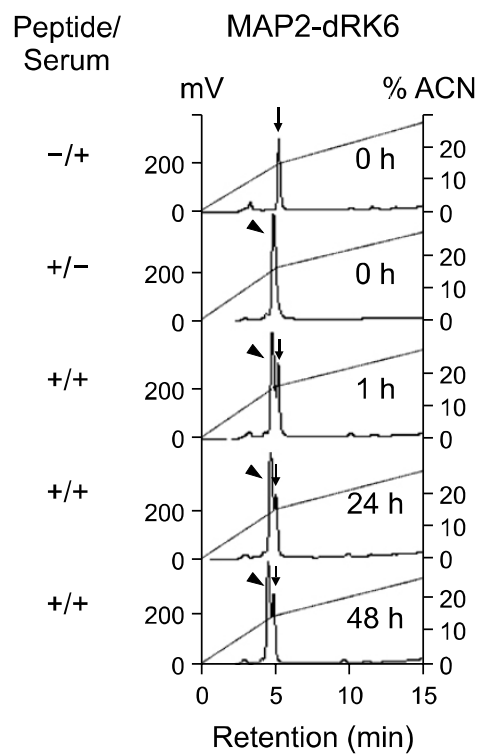

Figure 2. Inhibitory activity of MAP2-dRK6 on the binding of VEGF to HUVEC and its serum stability. (A) Binding of [ ${ }^{125}$ I]-VEGF 165 to HUVECs in the presence of each peptide was determined as described in Methods. Nonspecific binding of VEGF to HUVECs was less than 1\% of positive control. (B) The serum stability of MAP2-RK6, composed of L-peptides, and MAP2-dRK6, composed of D-peptides. Peptides were incubated with rat serum at $37^{\circ} \mathrm{C}$, and the mixture was fractionated by reverse phase HPLC as described in Methods. Peaks for serum ( $\downarrow$ ) and the peptides ( $\boldsymbol{\nabla})$ are indicated. The identity of MAP2-RK6 and MAP2-dRK6 was determined by mass spectrometry. ACN, acetonitrile.

lysine in the lysine- $\beta$-alanine branching unit. To evaluate which peptide has more potent anti-VEGF activity, we investigated the effects of those peptides on the binding of VEGF to their receptors on endothelial cells. The branched peptides, MAP2-RK6 and MAP2-dRK6, were more effective in the inhibition of VEGF binding to receptors than the non-branched ones, RK6 and dRK6 (Figure 2A).

Next, we compared the stability of the two branched peptides in serum. MAP2-dRK6 showed higher serum stability than MAP2-RK6; MAP2-dRK6 was stable for $48 \mathrm{~h}$ whereas MAP2-RK6 was degraded after $14 \mathrm{~h}$ (Figure 2B). This result is consistent with the previous reports (Hamamoto et al., 2002; Yoo et al., 2005), in which peptides with D-amino acids are more stable in serum than the peptides composed of L-amino acids due to their resistance to enzymatic hydrolysis. Therefore, we chose MAP2-dRK6 which has more potent anti-VEGF activity with enhanced serum stability for further experiments and selected dRK6 as a control peptide.

\section{MAP2-dRK6 inhibits VEGF-induced proliferation, ERK activation, migration, and tube formation of human endothelial cells}

To examine whether MAP2-dRK6 affects the actions of VEGF on endothelial cells, we investigated the effect of the peptide on VEGF-induced mitogenic and migratory activity on endothelial cells. MAP2-dRK6 inhibited the VEGF-induced incorporation of $\left[{ }^{3} \mathrm{H}\right]$-thymidine into DNA in human umbilical vein endothelial cells (HUVECs) more significantly than dRK6 (Figure 3A) without cytotoxicity (data not shown). Moreover, the anti-proliferative effect of MAP2-dRK6 was VEGF-specific as the peptide did not hinder the proliferation of HUVECs induced by basic fibroblast growth factor (bFGF). These results suggest that the inhibition was not a consequence of the positive charge of MAP2-dRK6 as the peptide did not inhibit the proliferation of endothelial cells by bFGF which like VEGF 165 requires negatively charged heparin to bind to its receptor and induce proliferation of the cells. We next investigated the effect of MAP2-dRK6 on VEGF-induced ERK signaling, which mainly contributes to VEGF-induced proliferation of endothelial cells. MAP2-dRK6 also inhibited the VEGF-induced ERK activation in a dose-dependent manner, whereas dRK6 showed no inhibitory activity (Figure 3B). We further investigated the inhibitory activity of MAP2-dRK6 on VEGF-induced migration and tube formation of endothelial cells. MAP2-dRK6 more significantly inhibited the migration of HUVECs than dRK6 in a dose-dependent manner (Figure 3C). Moreover, the inhibitory 
A
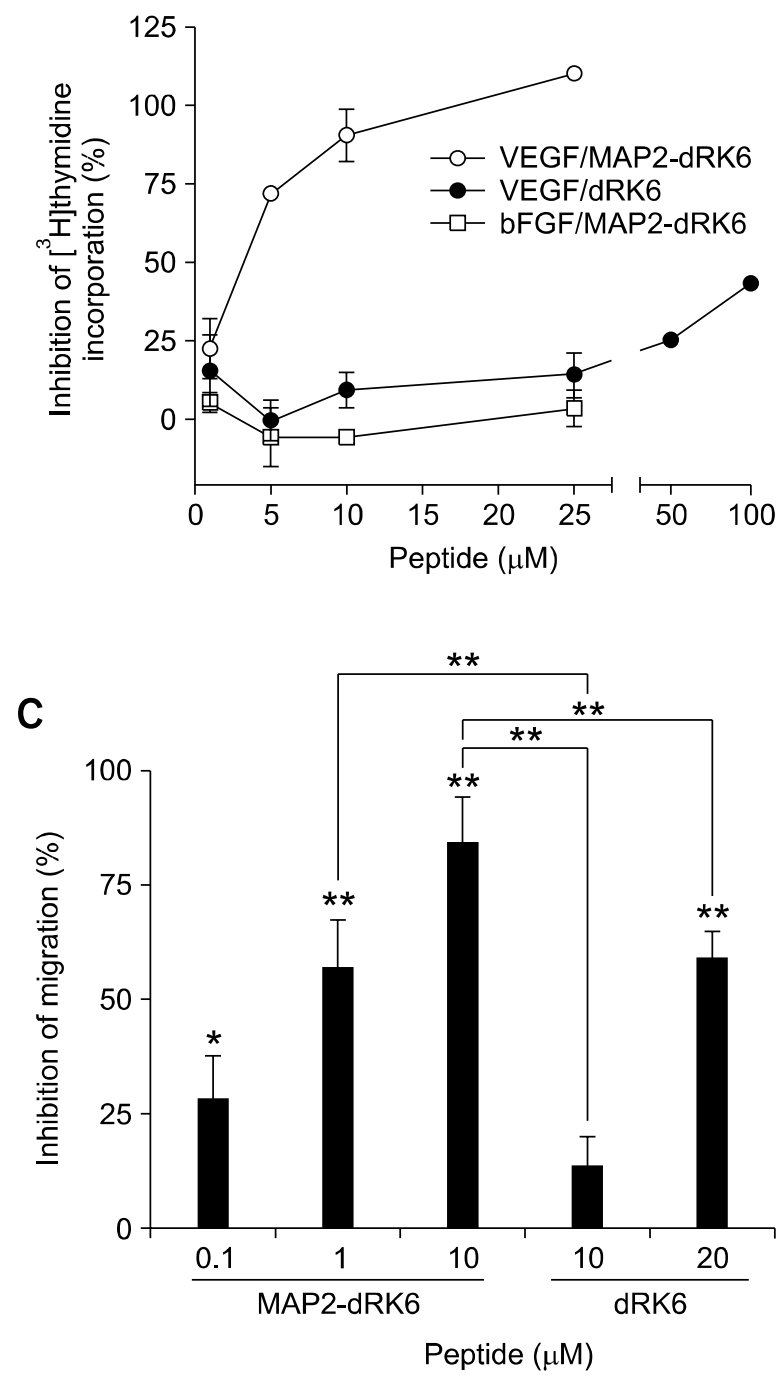

B

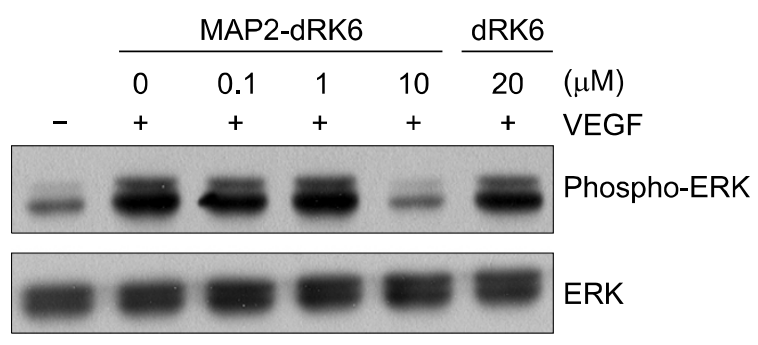

D

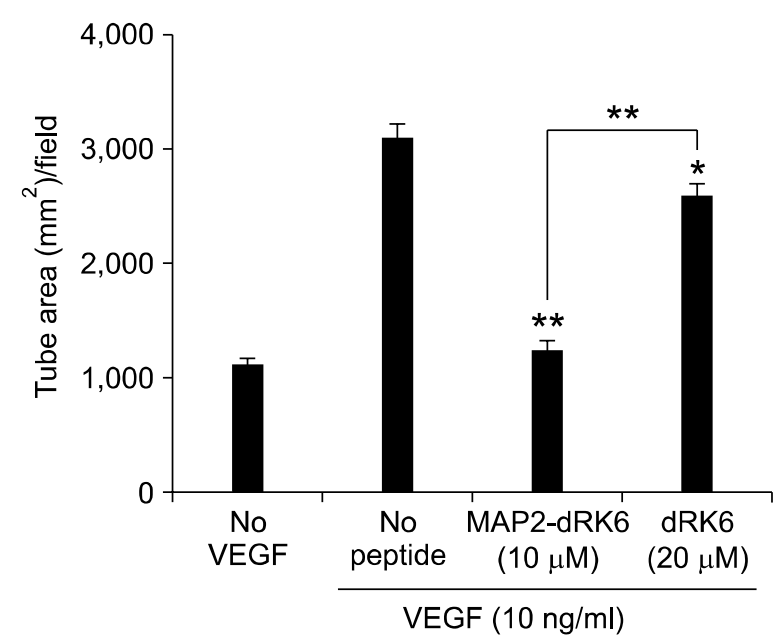

Figure 3. Inhibition of VEGF-induced proliferation, ERK activation, migration, and tube formation of endothelial cells by MAP2-dRK6. (A) The inhibitory activity of MAP2-dRK6 and dRK6 on VEGF-induced HUVEC proliferation. The proliferation of HUVECs was assessed by VEGF ( $5 \mathrm{ng} / \mathrm{ml})$-induced incorporation of [methyl- ${ }^{3} \mathrm{H}$ ]-thymidine into DNA in the presence of varying concentrations of MAP2-dRK6 or dRK6 as described in Methods. The effect of MAP2-dRK6 on bFGF (25 ng/ml)-induced proliferation was also investigated. (B) Effect of MAP2-dRK6 on VEGF-induced ERK signaling in HUVECs. HUVECs were stimulated by VEGF $(10 \mathrm{ng} / \mathrm{ml})$ in the presence of varying concentrations of MAP2-dRK6 and the proteins were separated by SDS gel electrophoresis. Western blotting was performed with anti-ERK and anti-phosphorylated ERK antibodies. (C) The inhibitory activity of MAP2-dRK6 and dRK6 on VEGF-induced HUVEC migration. HUVECs that migrated through the membrane (12 $\mu \mathrm{m}$ pore) were counted after incubation with VEGF (10 $\mathrm{ng} / \mathrm{ml}$ ) in the presence of varying concentrations of MAP2-dRK6. (D) The inhibitory activity of MAP2-dRK6 and dRK6 on VEGF-induced HUVEC tube formation. HUVECs grown on Matrigel-coated plate were treated with VEGF in the presence of MAP2-dRK6 or dRK6 were quantified as described in Methods. ${ }^{*}$ and ${ }^{* *}$ mean $P<0.05$ and $P<0.01$, respectively.

activity of MAP2-dRK6 on the tube formation of HUVECs was more potent than that of dRK6 (Figure 3D). These results obviously indicate that MAP2-dRK6 blocks angiogenesis in vitro more effectively than dRK6 by antagonizing VEGF.

As various tumor cells secrete VEGF that contributes to tumor angiogenesis (Senger et al., 1986), we evaluated the effect of MAP2-dRK6 on the endothelial cell proliferation and ERK signaling induced by conditioned medium derived from VEGF-secreting human colorectal cancer cells. The concentration of VEGF secreted from SW480 cells in the conditioned medium was $\sim 1.5 \mathrm{ng} / \mathrm{ml}$. We observed that MAP2-dRK6 effectively inhibited the proliferation and ERK signaling of HUVECs promoted by 2 -fold concentrated SW480 conditioned medium (Figures $4 \mathrm{~A}$ and $4 \mathrm{~B}$ ). These inhibitory activities were comparable to those of VEGF 
A

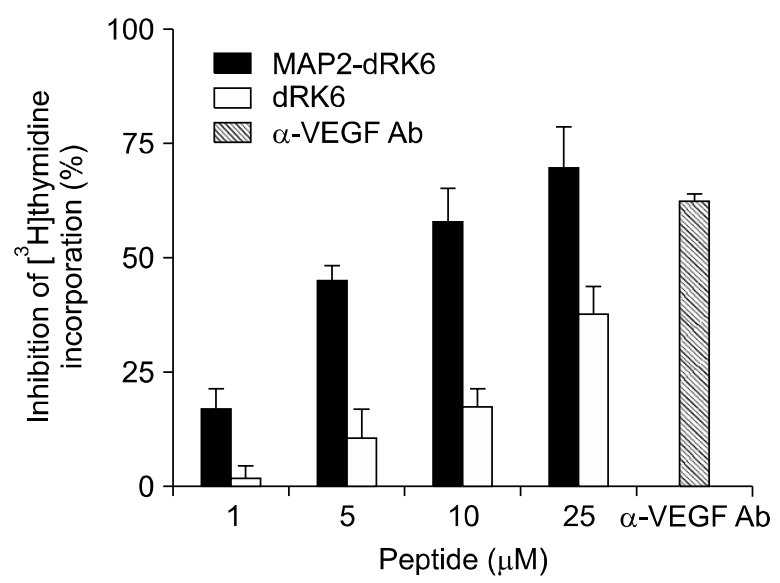

B

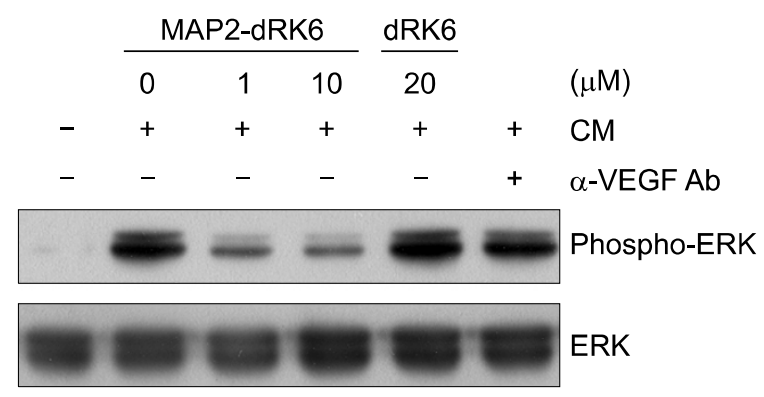

Figure 4. Effect of MAP2-dRK6 on endothelial cell proliferation and ERK activation induced by conditioned medium derived from VEGF-secreting human colorectal cancer cells. (A) The inhibitory activity of MAP2-dRK6 and dRK6 on the proliferation of HUVECs induced by tumor cell conditioned medium. The proliferation of HUVECs in response to SW480 conditioned medium was assessed by the incorporation of [methyl- ${ }^{3} \mathrm{H}$-thymidine into DNA in the presence of MAP2-dRK6 or dRK6 as described in Methods. (B) Effect of MAP2-dRK6 and dRK6 on ERK signaling in HUVECs induced by SW480 conditioned medium. CM, conditioned medium.

neutralizing antibody and much higher than those of dRK6. These results suggest that MAP2-dRK6 has a potent in vitro anti-angiogenic activity via neutralizing tumor-secreted VEGF bioactivity.

\section{MAP2-dRK6 blocks in vivo growth of VEGF-secreting colorectal cancer cells by the suppression of angiogenesis}

Since the acquisition of angiogenic phenotype is the decisive factor for tumor progression (Folkman et al., 1989; Hanahan and Folkman, 1996), we examined the effects of the peptides on the growth of SW480 cells xenografted in nude mice. MAP2-dRK6 ( $50 \mu \mathrm{g} /$ day) reduced the tumor volume by $58 \%$ and $65 \%$ at day 14 and 20 whereas dRK 6 reduced the tumor volume by $42 \%$ and $41 \%$ at day 14 and 20, respectively (Figure 5A). MAP2-dRK6 did not show any cytotoxicity or anti-proliferative activity on SW480 cells in vitro (data not shown), suggesting that the inhibitory activity is most likely through the anti-VEGF activity. Although anti-tumor growth activity of dRK6 is less potent than that of MAP2-dRK6, its activity is comparable to that of RK6 (Bae et al., 2000). Note that dRK6 has similar anti-VEGF activity as RK6 (Yoo et al., 2005).

To verify that this inhibition of tumor growth was due to the blockage of angiogenesis, we stained blood vessels in tumor tissues. MAP2-dRK6 strongly inhibited tumor-induced neovascularization; MAP2-dRK6 treatment more effectively decreased microvessel density within tumor when compared with untreated control or dRK6-treated group (Figure 5B). Next, we investigated if this blocking of angiogenesis could induce the apoptosis of tumor cells. As shown in Figure $5 \mathrm{C}$, MAP2-dRK6 induced the apoptosis of tumor cells and its activity was more potent than dRK6. Although we can not completely exclude other possibilities, our results strongly suggest that anti-VEGF peptide MAP2-dRK6 inhibits tumor growth by blocking angiogenesis induced by the tumor-secreted VEGF.

\section{Discussion}

In this study, we have developed a serum-stable branched form of VEGF-antagonizing peptide, MAP2-dRK6. The peptide has more potent anti-VEGF activity than the original peptides, RK6 and dRK6 (Bae et al., 2000; Yoo et al., 2005). MAP2-dRK6 inhibited in vitro angiogenic responses such as proliferation, ERK phosphorylation, migration, and tube formation of endothelial cells induced by VEGF and tumor conditioned medium more effectively than dRK6 through blocking VEGF binding to its cognate cell surface receptors. Moreover, MAP2-dRK6 blocks in vivo growth of VEGF-secreting colorectal cancer cells by the suppression of angiogenesis and by the subsequent induction of tumor cell apoptosis.

Peptide molecules play important roles in various biological phenomena. The use of peptide and its derivatives for the application to basic and medicinal research has been extensively studied 
A

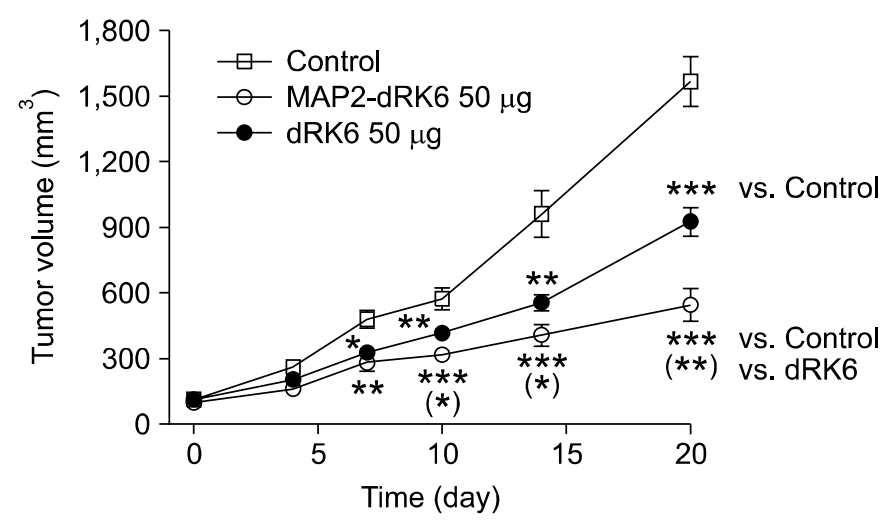

B
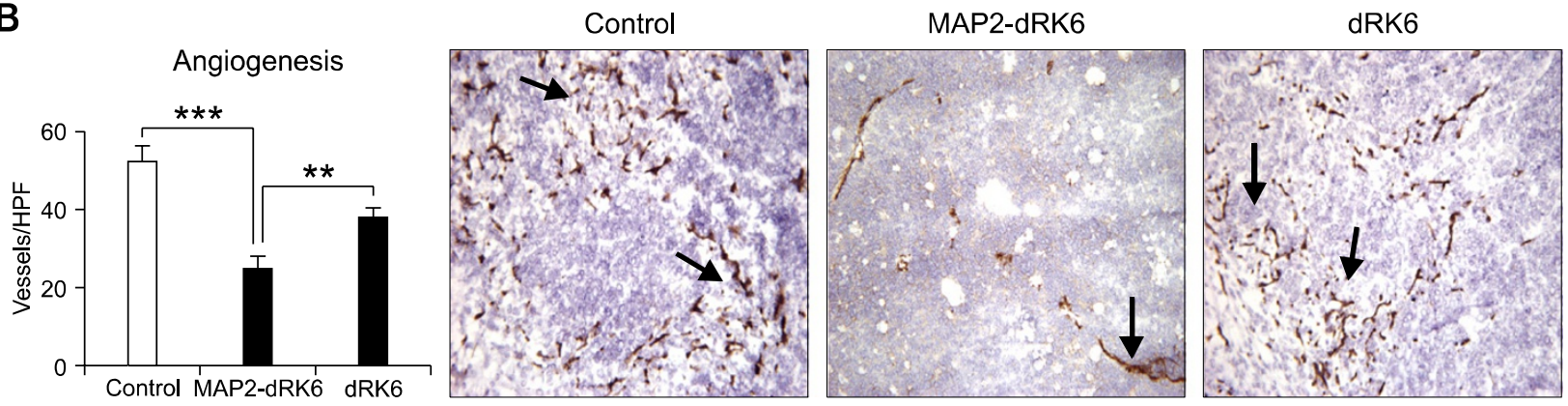

C

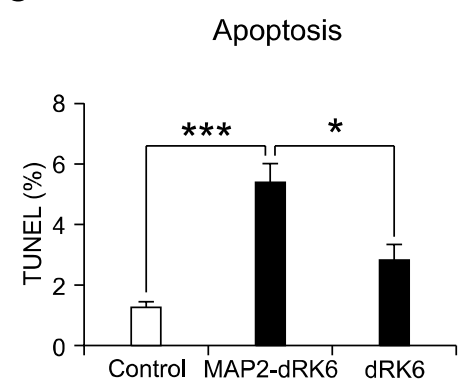

Control

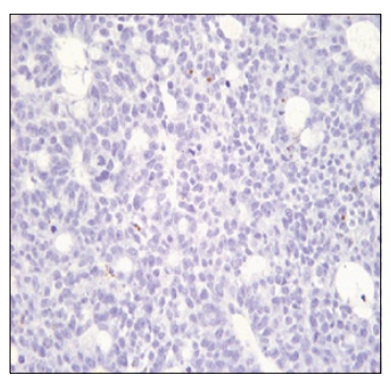

MAP2-dRK6

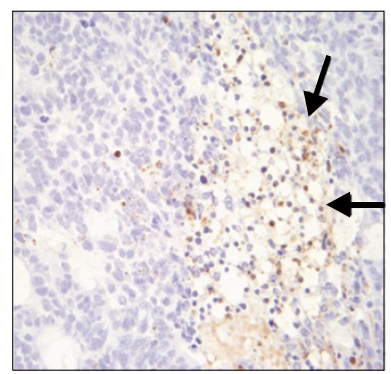

dRK6

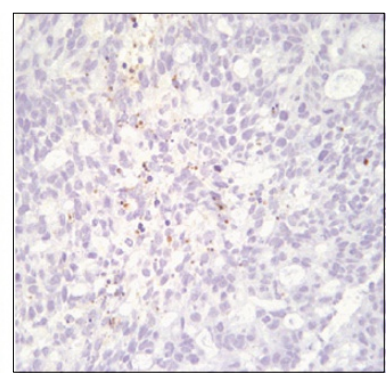

Figure 5. Inhibition of tumor growth through blocking angiogenesis by MAP2-dRK6. (A) Effect of MAP2-dRK6 and dRK6 on tumor growth in nude mice. After tumor volume reached $\sim 100 \mathrm{~mm}^{3}$, peptide $(50 \mu \mathrm{g})$ or saline control solution was injected subcutaneously and tumor volume was determined as described in Methods. (B) Effect of the peptides on tumor angiogenesis. Tumor tissues were stained with antibodies against endothelial cell-specific molecule (PECAM-1) and quantified. Arrows indicate the blood vessels. (C) Effect of the peptides on the apoptosis of tumor cells. Apoptotic cells are indicated by arrows. ${ }^{*}$, ${ }^{* *}$, and ${ }^{* * *}$ mean $P<0.05, P<0.01$, and $P<0.001$, respectively.

(Bruckdorfer et al., 2004; Park et al., 2009). Synthetic combinatorial peptide libraries have been utilized successfully to discover bioactive peptides such as antimicrobial peptides (Blondelle et al., 1996), ligands for cell surface receptors (Bae et al., 2003), protein kinase inhibitors and substrates (Wu et al., 1994; Songyang et al., 1995), and peptide mimotopes of receptor binding sites (Bracci et al., 2001). Moreover, a variety of modification methods have been developed and successfully applied to increase the activity and serum stability of the original peptides such as pegylation and acetylation (Sahu et al., 2000; Lee et al., 2005). For example, pegylation improved the pharmacological profile of glucagon-like peptide-1 (Lee et al., 2005), and acetylation increased the activity of compstatin through resistance to enzymatic degradation (Sahu et al., 2000).

MAP system was initially introduced to enhance the antigenicity of peptides to be used as vaccines (Tam, 1988). Besides its original function, the effectiveness of MAP with respect to corresponding 
monomeric peptide was also elucidated in the peptide inhibitors of protein-protein interactions (Bracci et al., 2002; Pini et al., 2006). Especially, multimeric forms of laminin B1 peptide showed improved biological activity compared to the original peptide with enhanced tumor growth and metastasis inhibition (Nomizu et al., 1993; Iwamoto et al., 1996). These observations suggest that the modification approach using MAP system may be useful in improving the activity of an anti-tumor peptide. MAP2-dRK6 has a lysine- $\beta$-alanine branching unit with two molecules of dRK6 attached. The VEGF-antagonizing activity of MAP2-dRK6 is several magnitudes higher than the activity expected from two molecules of dRK6. It is possible that the divalent structure of MAP2-dRK6 enables simultaneous interactions with VEGF dimer more effectively than dRK6 monomer, resulting in the amplification of the inhibitory activity. In fact, by virtue of its dendritic structure, MAP can make polyvalent interactions through associating with two or more ligands and receptors (Tam, 1988; Sadler and Tam, 2002). Such properties have been utilized for the development of more potent therapeutic agents (Nomizu et al., 1993; Iwamoto et al., 1996). On the other hand, a potential shortcoming of MAP system is that it might elicit antigenicity as it was originally developed to enhance the antigenicity of a peptide. However, we found that MAP2-dRK6 was not antigenic when injected intraperitoneally to mouse (data not shown).

Tumor-induced angiogenesis plays important roles in tumor growth and metastasis. Among the pro-angiogenic factors, the expression of VEGF has been reported to be strongly correlated with increased vascularity of tumor, advanced disease, and a worse prognosis (Takahashi et al., 1995, 1997). VEGF binding to its receptors expressed on endothelial cells transduces signals to induce proliferation (Ferrara and Henzel, 1989) and migration (Waltenberger et al., 1994) of endothelial cells, resulting in the promotion of angiogenesis. Thus, disruption of VEGF signaling through VEGF receptors can be the prospective method for blocking angiogenesis, tumor growth and metastasis. Many scientists have been and still are working on the development of effective anti-tumor drugs such as protein VEGF inhibitors. Bevacizumab, the first approved humanized variant of monoclonal antibody against VEGF (Presta et al., 1997; Ferrara et al., 2004), DC101, another monoclonal antibody targeting mouse VEGFR2 (Prewett et al., 1999), and VEGF-trap, a modified soluble VEGF receptor (Byrne et al., 2003), all significantly inhibited tumor growth in various cancer models. The efficacy of MAP2-dRK6 in the inhibition of tumor growth is comparable with protein VEGF inhibitors aforementioned in spite of infant stage of development. In addition, MAP2-dRK6 has an advantage of being synthesized more readily and in a larger amount than those protein inhibitors. Moreover, the extended half-life of MAP2-dRK6 may overcome the main drawback of peptide drugs which is short half-life due to proteolysis. Therefore, MAP2-dRK6 affords high possibility to be utilized as a potent lead compound, so that when used in combination with other therapeutic approaches would greatly increase the efficacy of the anti-tumor therapy. Furthermore, modification of MAP2-dRK6 to strengthen in vivo activity, pharmacokinetics, and safety profiles would possibly open its way to a favorable candidate for therapeutic agent in many excessive angiogenesis-related disorders such as rheumatoid arthritis, diabetic retinopathy, atherosclerosis, and especially various tumors.

\section{Methods}

\section{Materials}

All peptides used in this study were synthesized by Peptron, Inc. (Daejeon, Korea) and the purity of each peptide was over 95\%. Recombinant human VEGF and bFGF were purchased from R\&D systems (Minneapolis, $M N)$. Anti-VEGF antibody was obtained from R\&D systems and anti-ERK and phosphos-ERK antibodies were from Cell Signaling Technology (Danvers, MA).

\section{Cell culture and preparation of tumor conditioned medium}

HUVECs were cultured in Medium 199 (M199, Invitrogen, Carlsbad, CA) containing $20 \%$ of FBS, $5 \mathrm{ng} / \mathrm{ml}$ of bFGF, 50 $\mu \mathrm{g} / \mathrm{ml}$ of heparin (Sigma-Aldrich, St. Louis, MO), and $6 \mu \mathrm{g} / \mathrm{ml}$ of endothelial cell growth supplement (Sigma-Aldrich). SW480 human colorectal cancer cells were cultured in DMEM (Invitrogen) containing $10 \%$ of FBS. The conditioned medium was collected from SW480 cells by centrifugation for $20 \mathrm{~min}$ at $4^{\circ} \mathrm{C}$ and $3,000 \mathrm{~g}$ after incubation in serum-free DMEM for $48 \mathrm{~h}$.

\section{Binding of $\left[{ }^{125} \mid\right]-V E G F$ to VEGF receptors on the surface of HUVECs}

Binding assay between labeled VEGF and HUVECs was performed as described (Bae et al., 2000). HUVECs were seeded in a gelatin-coated 48 -well plate $\left(4 \times 10^{4}\right.$ cells/well). Nonspecific binding sites on HUVECs were blocked with $200 \mu \mathrm{l}$ of HUVEC binding assay medium (M199/25 mM HEPES/0.1\% BSA, pH 7.4) for $1 \mathrm{~h}$. $\left[{ }^{125} \mathrm{l}\right]$-VEGF $(20 \mathrm{nCi})$ was mixed with each peptide and preincubated at $4^{\circ} \mathrm{C}$ for $1 \mathrm{~h}$. HUVECs were treated with the mixture and incubated at $4^{\circ} \mathrm{C}$ for $3 \mathrm{~h}$. After the cells were 
solubilized with lysis buffer $(20 \mathrm{mM}$ Tris- $\mathrm{HCl} / 1 \%$ Triton $\mathrm{X}-100, \mathrm{pH} 7.4)$, the radioactivity of receptor-bound $\left.{ }^{125} \mathrm{I}\right]$-VEGF was determined in a gamma counter.

\section{Stability of MAP2-RK6 and MAP2-dRK6 in rat serum}

Stability of the peptides was investigated as described (Yoo et al., 2005). Serum was prepared from 16-week-old male Sprague Dawley rat. For estimating the stability of each peptide in serum, MAP2-RK6 or MAP2-dRK6 $(100 \mu \mathrm{g}$ in $50 \mu \mathrm{l}$ PBS) was incubated with $50 \mu$ of filtered rat serum at $37^{\circ} \mathrm{C}$ for the indicated time. The incubated sample was fractionated by $\mathrm{C} 18$ reverse phase HPLC with linear gradient of acetonitrile $\left(0.1 \%\right.$ trifluoroacetate in $\mathrm{H}_{2} \mathrm{O}$ for equilibration, and $0.1 \%$ trifluoroacetate in acetonitrile for elution). The identity of MAP2-RK6 and MAP2-dRK6 was determined by mass spectrometry.

\section{Endothelial cell proliferation assay}

VEGF $(5 \mathrm{ng} / \mathrm{ml})$, bFGF $(25 \mathrm{ng} / \mathrm{ml}) /$ heparin $(50 \mu \mathrm{g} / \mathrm{ml})$, or SW480 conditioned medium in HUVEC proliferation assay medium (M199/5\% FBS/10 mM HEPES, pH 7.4) was mixed and preincubated with each peptide at $37^{\circ} \mathrm{C}$ for $1 \mathrm{~h}$. The mixture was added to HUVECs seeded in a gelatin-coated 96 -well plate $\left(5 \times 10^{3}\right.$ cells/well), and the culture plate was incubated at $37^{\circ} \mathrm{C}$ for 1.5 days. To each well, [methyl- ${ }^{3} \mathrm{H}$ ]-thymidine $(0.5 \mu \mathrm{Ci})$ was added. After additional $12 \mathrm{~h}$ incubation, cells were washed and solubilized with $0.4 \mathrm{~N}$ of $\mathrm{NaOH}$ and neutralized with $2 \mathrm{~N} \mathrm{HCl}$. The radioactivity of incorporated $\left[{ }^{3} \mathrm{H}\right]$-thymidine was determined in a liquid scintillation counter.

\section{ERK signaling assay}

HUVECs were stimulated with VEGF $(10 \mathrm{ng} / \mathrm{ml}) /$ peptide or SW480 conditioned medium /peptide mixture for $10 \mathrm{~min}$ Total proteins were collected using M-PER (Thermo Scientific, Rockford, IL), separated by $10 \%$ SDS-PAGE, and transferred to a PVDF membrane. The blocked membrane was then incubated with the indicated antibodies, and the immunoreactive bands were visualized using a chemiluminescent substrate. The concentration of anti-VEGF antibody for neutralization of VEGF used in the experiments was $100 \mathrm{ng} / \mathrm{ml}$.

\section{Endothelial cell migration assay}

HUVEC migration assay was performed in a 48-well microchemotaxis chamber (Neuro Probe, Gaithersburg, MD) as described (Kim et al., 2002). Polycarbonate membrane with $12 \mu \mathrm{m}$ pores was coated with $0.1 \%$ gelatin solution for $5 \mathrm{~min}$ and dried. The bottom chamber was loaded with HUVECs $\left(3 \times 10^{4}\right.$ cells $)$ and the membrane was laid over the cells. The microchamber was then inverted and incubated at $37^{\circ} \mathrm{C}$ for $2 \mathrm{~h}$. After reinverting the chamber to upright position, the upper wells were loaded with VEGF $(10 \mathrm{ng} / \mathrm{ml}) /$ peptide mixture. The chamber was reincubated at $37^{\circ} \mathrm{C}$ for $4 \mathrm{~h}$ and then the membrane was fixed and stained using Diff-Quick solution (Baxter Healthcare, Deerfield, IL). The cells that migrated through the filter membrane were quantified by counting three random fields of each well.

\section{Capillary-like tube formation assay}

Tube formation assay was performed as described previously (Kim et al., 2002). HUVEC were plated in a GFR Matrigel (BD Biosciences, San Jose, CA)-coated 24-well plate $\left(2 \times 10^{4}\right.$ cells $/$ well $)$ and then VEGF $(10 \mathrm{ng} / \mathrm{ml}) /$ peptide mixture was added. After $4 \mathrm{~h}$ incubation, three randomly chosen fields were photographed at 10 -fold magnification. Total tube areas in the images were measured using Scion Image program.

\section{Tumor growth inhibition assay in nude mice}

Tumor growth inhibition assay in nude mice was performed as described (Bae et al., 2000). Viable SW480 cells $(5 \times$ $10^{6}$ cells) prepared in serum-free DMEM were injected subcutaneously over the right scapular region of pathogen-free, four-week-old male athymic nude mice (BALB/c/nu/nu, SLC, Japan). From the establishment of tumor ( $\sim 100 \mathrm{~mm}^{3}$ in tumor volume), mice received subcutaneous injection of each peptide $(50 \mu \mathrm{g})$ or control normal physiologic saline daily for 14 days. Tumor volumes were measured and determined using the following formula: Volume $=0.5 \times(\text { width })^{2} \times$ length. Each experimental group was consisted of six animals.

\section{Immunohistochemistry}

Immunohistochemistry of tumor mass was performed as described (Yoon et al., 2004). After mice were sacrificed at day 20 of peptide injection, tumors were removed and bisected. One part of the tumor was placed in formalin for paraffin block preparation, and the other part was frozen for cryocut sections. To immunolocalize tumor blood vessels, cryosections were stained with a rat anti-mouse CD31 antibody (BD PharMingen, San Diego, CA). Visualization of the antigen-antibody reaction was carried out using an anti-rat immunoglobulin horseradish peroxidase detection kit (BD PharMingen). Vessel density was determined by counting the stained vessels at $200 \times$. The degree of apoptosis was evaluated using an ApopTag apoptosis detection kit (Intergen, Norcross, GA). The apoptotic index was calculated as the percentage of nuclei stained by peroxidase and showing nuclear halo or apoptotic bodies. Positive cells were counted among a minimum of 400 cells/histological section at $400 \times$. All animal experiments were done in accordance with the guidelines issued by Chungnam National University about the ethics of animal experimentation.

\section{Statistical analysis}

Values of results were expressed as mean and standard deviation or standard error mean, and the significance was established by Student's $t$ test. In all analyses, the level of statistical significance was more than $95 \%$ confidence level $(P<0.05) .{ }^{*},{ }^{* *}$, or ${ }^{* * *}$ means $P$ value less than $0.05,0.01$, or 0.001 , respectively. 


\section{Acknowledgements}

This work was supported by Mid-career Researcher Program through NRF grant funded by the MEST (No.2009-0080709) and the Korean Research Foundation Grant funded by the Korean Government (MOEHRD, Basic Research Promotion Fund) (KRF-2007-313-C00716).

\section{References}

Aiello LP, Pierce EA, Foley ED, Takagi $\mathrm{H}$, Chen $\mathrm{H}$, Riddle L, Ferrara N, King GL, Smith LE. Suppression of retinal neovascularization in vivo by inhibition of vascular endothelial growth factor (VEGF) using soluble VEGF-receptor chimeric proteins. Proc Natl Acad Sci USA 1995; 92:10457-61

Bae DG, Gho YS, Yoon WH, Chae CB. Arginine-rich anti-vascular endothelial growth factor peptides inhibit tumor growth and metastasis by blocking angiogenesis. J Biol Chem 2000;275:13588-96

Bae YS, Park EY, Kim Y, He R, Ye RD, Kwak JY, Suh PG, Ryu $\mathrm{SH}$. Novel chemoattractant peptides for human leukocytes. Biochem Pharmacol 2003;66:1841-51

Blondelle SE, Perez-Paya E, Houghten RA. Synthetic combinatorial libraries: novel discovery strategy for identification of antimicrobial agents. Antimicrob Agents Chemother 1996;40:1067-71

Borgstrom P, Hillan KJ, Sriramarao P, Ferrara N. Complete inhibition of angiogenesis and growth of microtumors by anti-vascular endothelial growth factor neutralizing antibody: novel concepts of angiostatic therapy from intravital videomicroscopy. Cancer Res 1996;56:4032-9

Bracci L, Lozzi L, Lelli B, Pini A, Neri P. Mimotopes of the nicotinic receptor binding site selected by a combinatorial peptide library. Biochemistry 2001;40:6611-9

Bracci L, Lozzi L, Pini A, Lelli B, Falciani C, Niccolai N, Bernini A, Spreafico A, Soldani P, Neri P. A branched peptide mimotope of the nicotinic receptor binding site is a potent synthetic antidote against the snake neurotoxin alpha-bungarotoxin. Biochemistry 2002;41:10194-9

Bruckdorfer T, Marder O, Albericio F. From production of peptides in milligram amounts for research to multi-tons quantities for drugs of the future. Curr Pharm Biotechnol 2004;5:29-43

Byrne AT, Ross L, Holash J, Nakanishi M, Hu L, Hofmann JI, Yancopoulos GD, Jaffe RB. Vascular endothelial growth factor-trap decreases tumor burden, inhibits ascites, and causes dramatic vascular remodeling in an ovarian cancer model. Clin Cancer Res 2003;9:5721-8

Carmeliet P. Mechanisms of angiogenesis and arteriogenesis. Nat Med 2000;6:389-95

Carmeliet P, Jain RK. Angiogenesis in cancer and other diseases. Nature 2000;407:249-57

de Vries C, Escobedo JA, Ueno H, Houck K, Ferrara N, Williams LT. The fms-like tyrosine kinase, a receptor for vascular endothelial growth factor. Science 1992;255:
989-91

Ferrara N, Henzel WJ. Pituitary follicular cells secrete a novel heparin-binding growth factor specific for vascular endothelial cells. Biochem Biophys Res Commun 1989;161: 851-8

Ferrara N, Hillan KJ, Gerber HP, Novotny W. Discovery and development of bevacizumab, an anti-VEGF antibody for treating cancer. Nat Rev Drug Discov 2004;3:391-400

Folkman J, Watson K, Ingber D, Hanahan D. Induction of angiogenesis during the transition from hyperplasia to neoplasia. Nature 1989;339:58-61

Hamamoto K, Kida Y, Zhang Y, Shimizu T, Kuwano K. Antimicrobial activity and stability to proteolysis of small linear cationic peptides with D-amino acid substitutions. Microbiol Immunol 2002;46:741-9

Hanahan D, Folkman J. Patterns and emerging mechanisms of the angiogenic switch during tumorigenesis. Cell 1996;86:353-64

Iwamoto Y, Nomizu M, Yamada Y, Ito Y, Tanaka K, Sugioka $Y$. Inhibition of angiogenesis, tumour growth and experimental metastasis of human fibrosarcoma cells HT1080 by a multimeric form of the laminin sequence Tyr-Ile-Gly-Ser-Arg (YIGSR). Br J Cancer 1996;73:589-95

Kim CW, Lee HM, Lee TH, Kang C, Kleinman HK, Gho YS. Extracellular membrane vesicles from tumor cells promote angiogenesis via sphingomyelin. Cancer Res 2002;62: 6312-7

Kim KJ, Li B, Houck K, Winer J, Ferrara N. The vascular endothelial growth factor proteins: identification of biologically relevant regions by neutralizing monoclonal antibodies. Growth Factors 1992;7:53-64

Kondo S, Asano M, Matsuo K, Ohmori I, Suzuki H. Vascular endothelial growth factor/vascular permeability factor is detectable in the sera of tumor-bearing mice and cancer patients. Biochim Biophys Acta 1994;1221:211-4

Lee SH, Lee S, Youn YS, Na DH, Chae SY, Byun Y, Lee KC. Synthesis, characterization, and pharmacokinetic studies of PEGylated glucagon-like peptide-1. Bioconjug Chem 2005; 16:377-82

Liotta LA, Steeg PS, Stetler-Stevenson WG. Cancer metastasis and angiogenesis: an imbalance of positive and negative regulation. Cell 1991;64:327-36

Millauer B, Wizigmann-Voos S, Schnurch $\mathrm{H}$, Martinez $\mathrm{R}$, Moller NP, Risau W, Ullich A. High affinity VEGF binding and developmental expression suggest Flk-1 as a major regulator of vasculogenesis and angiogenesis. Cell 1993;72: 835-46

Nomizu M, Yamamura K, Kleinman HK, Yamada Y. Multimeric forms of Tyr-Ile-Gly-Ser-Arg (YIGSR) peptide enhance the inhibition of tumor growth and metastasis. Cancer Res 1993;53:3459-61

Park K, Choi K, Kim H, Kim K, Lee MH, Lee JH, Kim Rim JC. Isoflavone-deprived soy peptide suppresses mammary tumorigenesis by inducing apoptosis. Exp Mol Med 2009;41:371-81 
Pini A, Runci Y, Falciani C, Lelli B, Brunetti J, Pileri S, Fabbrini M, Lozzi L, Ricci C, Bernini A, Tonello F, Dal Molin F, Neri P, Niccolai N, Bracci L. Stable peptide inhibitors prevent binding of lethal and oedema factors to protective antigen and neutralize anthrax toxin in vivo. Biochem J 2006;395:157-63

Presta LG, Chen H, O'Connor SJ, Chisholm V, Meng YG, Krummen L, Winkler M, Ferrara N. Humanization of an anti-vascular endothelial growth factor monoclonal antibody for the therapy of solid tumors and other disorders. Cancer Res 1997;57:4593-9

Prewett M, Huber J, Li Y, Santiago A, O'Connor W, King K, Overholser J, Hooper A, Pytowski B, Witte L, Bohlen P, Hicklin DJ. Antivascular endothelial growth factor receptor (fetal liver kinase 1) monoclonal antibody inhibits tumor angiogenesis and growth of several mouse and human tumors. Cancer Res 1999;59:5209-18

Sadler K, Tam JP. Peptide dendrimers: applications and synthesis. J Biotechnol 2002;90:195-229

Sahu A, Soulika AM, Morikis D, Spruce L, Moore WT, Lambris JD. Binding kinetics, structure-activity relationship, and biotransformation of the complement inhibitor compstatin. J Immunol 2000;165:2491-9

Senger DR, Perruzzi CA, Feder J, Dvorak HF. A highly conserved vascular permeability factor secreted by a variety of human and rodent tumor cell lines. Cancer Res 1986;46:5629-32

Songyang Z, Carraway KL, 3rd, Eck MJ, Harrison SC, Feldman RA, Mohammadi M, Schlessinger J, Hubbard SR, Smith DP, Eng C, et al. Catalytic specificity of protein-tyrosine kinases is critical for selective signalling. Nature 1995;373:536-9

Strawn LM, McMahon G, App H, Schreck R, Kuchler WR, Longhi MP, Hui TH, Tang C, Levitzki A, Gazit A, Chen I, Keri G, Orfi L, Risau W, Flamme I, Ullrich A, Hirth KP, Shawver LK.
Flk-1 as a target for tumor growth inhibition. Cancer Res 1996;56:3540-5

Takahashi Y, Kitadai Y, Bucana CD, Cleary KR, Ellis LM. Expression of vascular endothelial growth factor and its receptor, KDR, correlates with vascularity, metastasis, and proliferation of human colon cancer. Cancer Res 1995;55:3964-8

Takahashi Y, Tucker SL, Kitadai Y, Koura AN, Bucana CD, Cleary KR, Ellis LM. Vessel counts and expression of vascular endothelial growth factor as prognostic factors in node-negative colon cancer. Arch Surg 1997;132:541-6

Tam JP. Synthetic peptide vaccine design: synthesis and properties of a high-density multiple antigenic peptide system. Proc Natl Acad Sci USA 1988;85:5409-13

Waltenberger J, Claesson-Welsh L, Siegbahn A, Shibuya M, Heldin $\mathrm{CH}$. Different signal transduction properties of KDR and Flt1, two receptors for vascular endothelial growth factor. J Biol Chem 1994;269:26988-95

Wu J, Ma QN, Lam KS. Identifying substrate motifs of protein kinases by a random library approach. Biochemistry 1994;33:14825-33

Yancopoulos GD, Davis S, Gale NW, Rudge JS, Wiegand SJ, Holash J. Vascular-specific growth factors and blood vessel formation. Nature 2000;407:242-8

Yoo SA, Bae DG, Ryoo JW, Kim HR, Park GS, Cho CS, Chae $\mathrm{CB}$, Kim WU. Arginine-rich anti-vascular endothelial growth factor (anti-VEGF) hexapeptide inhibits collagen-induced arthritis and VEGF-stimulated productions of TNF-alpha and IL-6 by human monocytes. J Immunol 2005;174:5846-55

Yoon WH, Jung YJ, Kim TD, Li G, Park BJ, Kim JY, Lee YC, Kim JM, Park JI, Park HD, No ZS, Lim K, Hwang BD, Kim YS. Gabexate mesilate inhibits colon cancer growth, invasion, and metastasis by reducing matrix metalloproteinases and angiogenesis. Clin Cancer Res 2004;10:4517-26 